\title{
HPLC with charged aerosol detector (CAD) as a quality control platform for analysis of carbohydrate polymers
}

\author{
Rajarshi Ghosh and Paul Kline* (D)
}

\begin{abstract}
Objective: QC analysis of carbohydrates has been historically cumbersome due to lengthy and laborious derivatization techniques and the requirement of complimentary instrumentation. HILIC-CAD has emerged as an effective platform for direct monosaccharide composition analysis of complex carbohydrates without derivatization. Although, several neutral sugars have been separated and detected using HILIC-CAD, there has not been any report on acidic and amino sugar analysis using this method. In this study, we developed a gradient method for simultaneous analysis of acidic, amino and select neutral monosaccharides. As an application of the HILIC-CAD method, we performed composition analysis of commercially purchased hyaluronic acid products. Additionally, since CAD is suitable for SEC experiments, we tested the homogeneity of hyaluronic acids using a SEC-CAD method.
\end{abstract}

Results: We separated common uronic acids (GlcA, GalA, LIdoA and Neu5Ac), amino sugars (GlcN, GalN and GlcNAc) and select neutral sugars (LRha, LFuc, Man and Gal) using a gradient HILIC-CAD method. The optimized gradient method demonstrated good linearity $\left(R^{2}>0.99\right)$, precision (RSD $\left.<8 \%\right), L O D(<85 \mathrm{ng} / \mathrm{mL}$ ) and LOQ $(<280 \mathrm{ng} / \mathrm{mL})$. HILIC-CAD analysis of commercially purchased hyaluronic acid products indicated that samples were composed of GlcNAc and GlcA. Additionally, SEC-CAD chromatograms indicated the heterogeneous nature of the samples.

Keywords: HILIC, HPSEC, QC of carbohydrates, Hyaluronic acid, Uronic acids, Amino sugars

\section{Introduction}

Carbohydrates have become increasingly important commercially not only as food or structural building blocks, but also as natural health products [1]. It is essential to have proper QC of carbohydrate-based therapeutics to ensure their efficacy and safety. Physicochemical properties such as homogeneity, size and composition serve as important QC parameters for carbohydrates [1]. The QC process is often time-consuming and expensive due to the lack of a single platform capable of analyzing necessary parameters. Analysts rely on laborious derivatization procedures and complementary analytical instrumentation to test products. These problems underline the

*Correspondence: paul.kline@mtsu.edu

Department of Chemistry, Middle Tennessee State University, 1301 E Main Street, MTSU Box 68, Murfreesboro, TN 37132, USA need for a simple multipurpose platform for rapid QC of carbohydrates.

Analysis of complex carbohydrates has been historically challenging due to their heterogeneity and diversity [1]. Additionally, monosaccharide composition analysis can be tricky due to the presence of epimers, formation of anomers and lack of a chromophore [2]. Common analytical techniques (GC-MS and reverse phase HPLC) for composition analysis require appropriate derivatization of monosaccharides [3, 4]. As a result analysis of underivatized monosaccharides is becoming increasingly popular. High performance anion exchange chromatography with pulsed amphoteric detection (HPAEC-PAD) has proven to be an effective tool for direct analysis of monosaccharides in recent years [5]. However, the high $\mathrm{pH}$ of the eluent resulting in epimerization and degradation of carbohydrates, unstable baseline, loss of sensitivity and requirement of a dedicated base-compatible HPLC are 
some of its disadvantages [6]. Hydrophilic interaction liquid chromatography (HILIC) coupled to evaporative light scattering detector (ELSD), mass spectrometer (MS), refractive index detector (RID) and charged aerosol detector (CAD) offer other alternate ways of analyzing underivatized carbohydrates [2, 7-10]. The CAD, introduced by Dixon and Peterson [11], offers several advantages compared to other detectors used in the direct analysis of sugars. The response of CAD does not depend on the structural properties of the analyte and it offers greater sensitivity than ELSD [12]. It is compatible with gradient elution and allows detection of all non-volatile and most semi-volatile analytes [13]. It is also relatively cheap and easy to use compared to MS. Hence, HILIC coupled with CAD can be an excellent tool for direct composition analysis and detection of impurities in samples. Although, neutral sugars have been previously separated using HILIC-CAD, there has not been any reports on the analysis of acidic and amino sugars.

In this study, a method has been developed to separate and detect amino sugars (GlcN, GalN and GlcNAc) and acidic sugars (GlcA, GalA, Neu5Ac and LIdoA) without derivatization using HILIC-CAD. Commonly found $\mathrm{N}$-linked neutral sugar residues in mammalian glycoproteins such as LFuc, Gal and Man were also simultaneously separated. As an application of our proposed QC platform, we analyzed the monomer composition of commercially available hyaluronic acid (HA) products.

CAD has also been shown to be effective in size exclusion chromatography (SEC) with better impurity and polydispersity profiles compared to RID ad ELSD [14]. Therefore, SEC-CAD can be potentially employed for homogeneity and molecular weight analysis of carbohydrates. In this study, we demonstrated the homogeneity of commercially purchased HA products using a SECCAD method adapted from Chen et al. [15].

\section{Main text \\ Materials and methods \\ Material and reagents}

All standards, solvents and buffer additives were of HPLC grade (Sigma Aldrich, Synthose Inc., and Fisher Scientific). HA supplements were purchased from local supermarkets.

\section{Separation of monosaccharides by HILIC-CAD}

A Dionex Ultimate 3000 HPLC system coupled to a Corona charged aerosol detector was used for the chromatographic analysis. Separation was carried out using a Waters XBridge BEH Amide XP $(3 \times 150 \mathrm{~mm} ; 2.5 \mu \mathrm{m})$ column. The mobile phase of the optimized method consisted of (A) $90 \%$ acetonitrile with $0.2 \%$ TEA and $25 \mathrm{mM}$ ammonium acetate; and (B) water with $0.2 \%$ TEA and $25 \mathrm{mM}$ ammonium acetate. The following gradient elution was used: $0 \% \mathrm{~B}$ at $0-15 \mathrm{~min}, 0-18 \% \mathrm{~B}$ at $15-40 \mathrm{~min}$, $18 \% \mathrm{~B}$ at $40-45 \mathrm{~min}, 18-0 \% \mathrm{~B}$ at $45-47 \mathrm{~min}$ and $0 \% \mathrm{~B}$ at $47-55 \mathrm{~min}$. The flow rate was $0.5 \mathrm{~mL} / \mathrm{min}$. A column temperature of $50{ }^{\circ} \mathrm{C}$ and an injection volume of $10 \mu \mathrm{L}$ (mixed standard monosaccharide solution at a concentration of $225 \mu \mathrm{g} / \mathrm{mL}$ ) was used for the analysis. The effects of column temperature $\left(50{ }^{\circ} \mathrm{C}, 40{ }^{\circ} \mathrm{C}\right.$ and $\left.30{ }^{\circ} \mathrm{C}\right)$ and buffers such as ammonium acetate $(25 \mathrm{mM}$ and $20 \mathrm{mM})$, ammonium formate $(25 \mathrm{mM}$ and $20 \mathrm{mM})$ and triethylamine (TEA) $(0.2 \%$ and $0.1 \%)$ on chromatographic separation were evaluated. The following parameters were used for the CAD detector: (i) nitrogen gas pressure: 35 psi; (ii) detector response $100 \mathrm{pA}$; and (iii) noise filter: high. Data processing was carried out using Chromeleon 6.8 software.

\section{Validation}

Seven different concentrations $(15-1000 \mu \mathrm{g} / \mathrm{mL})$ of the standards were injected in triplicate to construct a double logarithmic plot, which was used as a calibration curve [16]. The limit of detection (LOD) and limit of quantification (LOQ) was calculated based on previously reported methods $[16,17]$. Intra-day precision (\%RSD) was determined by repeating the analysis of a standard solution five times in a single day. Inter-day precision (\%RSD) analysis was performed over 3 days.

\section{Application of HPLC-CAD as a QC platform}

The monosaccharide composition of commercially purchased HA products were analyzed to demonstrate the application of the HILIC-CAD method. Samples (> $5 \mathrm{mg}$ ) were hydrolyzed using $2 \mathrm{~mL}$ of $2 \mathrm{M}$ trifluoroacetic acid (TFA) at $110{ }^{\circ} \mathrm{C}$ for $2 \mathrm{~h}$ prior to composition analysis.

The homogeneity of HA was also analyzed by a separate high performance size exclusion chromatography (HPSEC) experiment. GPC grade HA standards and commercially purchased samples were analyzed on a TOSOH TSKgel G4000PW xl $(7.8 \times 300$ mm; $10 \mu \mathrm{m})$ column for $35 \mathrm{~min}$. Ammonium acetate $(25 \mathrm{mM})$ at a flow rate of $0.5 \mathrm{~mL} / \mathrm{min}$ was used as the eluent. A volume of $10 \mu \mathrm{L}$ of sample was injected into the column. The CAD parameters were the same as before.

\section{Results and discussion}

\section{Separation of monosaccharides by HILIC-CAD}

The gradient method was able to separate uronic acids (GlcA, GalA, Neu5Ac and LIdoA) and amino sugars (GlcN, GalN and GlcNAc) as well as select neutral sugar residues (LRha, LFuc, Man and Gal) (Fig. 1). The deoxy monosaccharides eluted first followed by acetylated 


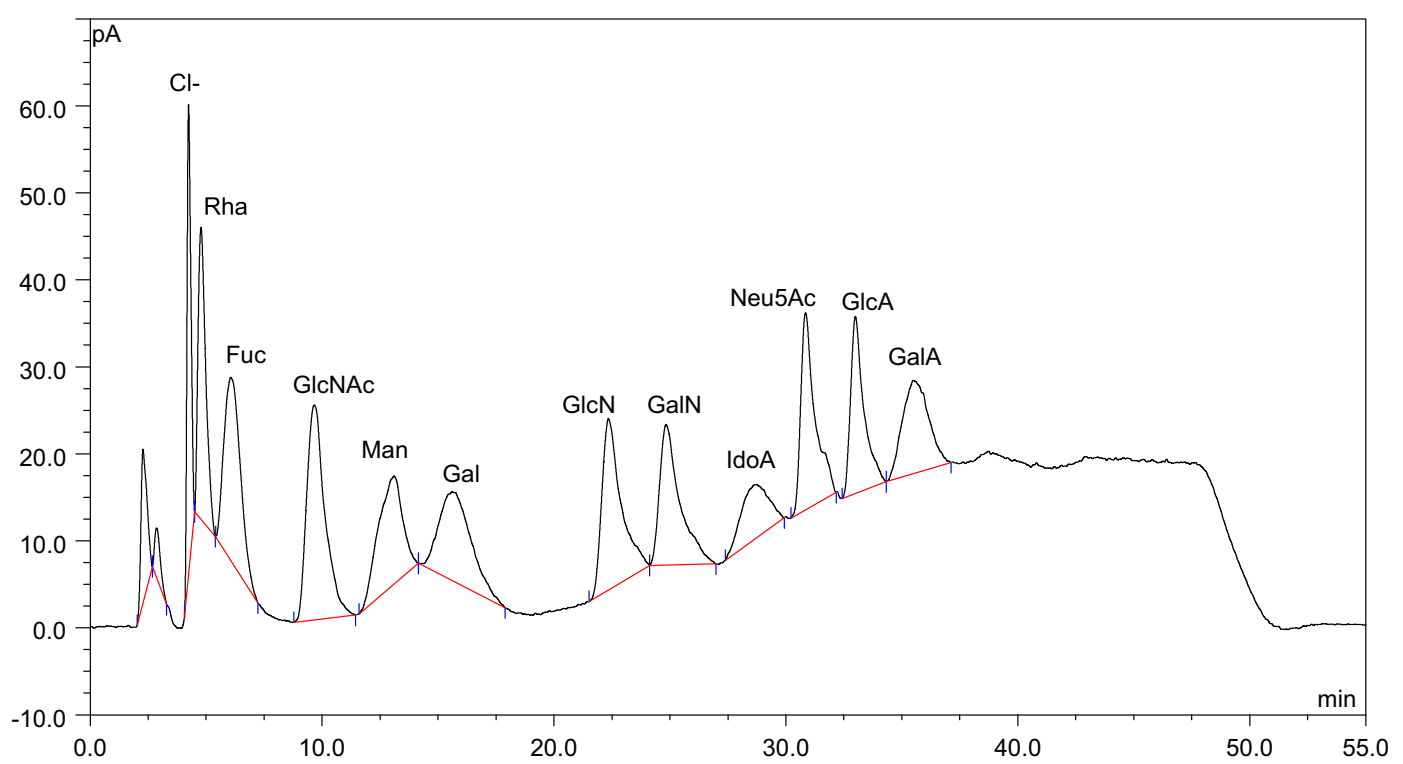

Fig. 1 Separation of monosaccharides by HILIC-CAD. The HILIC-CAD gradient method was able to separate a mixture of 11 monosaccharide standards (225 mg/mL) (Rha, Fuc, GlcNAc, Man, Gal, GlcN, GalN, IdoA, Neu5Ac, GlcA and GalA) in this study. Buffer additives of $25 \mathrm{mM}$ ammonium acetate and $0.2 \% \mathrm{TEA}$ and a column temperature of $50^{\circ} \mathrm{C}$ were used for the HPLC analysis

amino sugars, aldohexoses, amino sugars and uronic acids. This method can be potentially adapted for the monosaccharide composition analysis of other acidic/ amino sugar-rich polysaccharides such as glycosaminoglycans and N-linked sugar residues found in mammalian glycoproteins.

A major problem in analyzing monosaccharides is the formation of $\alpha$ and $\beta$ anomers resulting in split peaks. Addition of tertiary amines in the mobile phase, high temperature and high $\mathrm{pH}$ are common ways to increase the rate of the interconversion of anomers $[9,18]$. During method development, different column temperatures $\left(30{ }^{\circ} \mathrm{C}, 40{ }^{\circ} \mathrm{C}\right.$ and $50{ }^{\circ} \mathrm{C}$ ) (Additional file 1: Fig. S1) and concentrations of TEA in the mobile phase $(0.1 \%$ and $0.2 \%$ ) (Additional file 1: Fig. S2) were used to suppress split peaks. Although, temperatures below $30{ }^{\circ} \mathrm{C}$ were able to separate most monosaccharides, it produced anomeric peak doublets (data not shown). Column temperature of $50{ }^{\circ} \mathrm{C}$ was able to adequately separate acidic and amino sugars along with select neutral sugars without formation of split peaks (Fig. 1). Addition of TEA in the mobile phase was also found to enhance mutarotation rates to yield single peaks (Additional file 1: Fig. S2). A concentration of $0.1 \%$ TEA was found to be sufficient to suppress peak doublets for most monosaccharides except mannose (Additional file 1: Fig. S2). Hence, a combination of $0.2 \%$ TEA and $50{ }^{\circ} \mathrm{C}$ column temperature was chosen for the optimized method. Higher concentrations of TEA $(>0.2 \%)$ or high $\mathrm{pH}(>10)$ caused substantial detector noise and unstable baseline. Apart from tertiary amines, we also tested the effects of ammonium acetate (Additional file 1: Fig. S3) and ammonium formate (data not shown), on separation. Ammonium formate gave a slightly higher detector response compared to ammonium acetate (data not shown). However, ammonium acetate gave a more stable baseline and was subsequently chosen as the buffer of choice. Although, higher ammonium acetate concentrations increased retention times, it also resulted in better separations (Additional file 1: Fig. S3). Ammonium acetate concentrations above $25 \mathrm{mM}$ led to high detector noise.

The optimized method was validated by evaluating linearity, LOD, LOQ and intra and inter-day precision of peak areas (Additional file 1: Table S1). After double logarithmic transformation, the calibration curves of 11 analytes showed good linearity ( $>0.99)$. The LOD and LOQ values were in the range of $50-83 \mathrm{ng} / \mathrm{mL}$ and 170 $278 \mathrm{ng} / \mathrm{mL}$ respectively, which were higher compared to earlier studies involving neutral sugars $[9,16]$. The higher LOD and LOQs reported in this study might be due to the higher concentrations of ammonium acetate $(25 \mathrm{mM})$ and TEA $(0.2 \%)$ in the mobile phase. The results indicated satisfactory intra and inter-day precision of peak areas $(1-8 \%$ RSD). 


\section{HPLC-CAD as a QC platform}

The glycosaminoglycan HA, a widely used dietary supplement, was used to demonstrate the utility of HPLC-CAD as a QC platform. HA is composed of a repeating disaccharide unit made up of GlcNAc and GlcA [19]. The HILIC-CAD gradient method was used to analyze the composition of a HA standard and a commercially available HA product (Fig. 2). Figure 2a shows the chromatogram of GlcNAc and GlcA standards treated with $2 \mathrm{M}$ TFA at $110{ }^{\circ} \mathrm{C}$ for $2 \mathrm{~h}$. The breakdown of GlcNAc into GlcN is evident from the chromatogram. Figure $2 \mathrm{~b}$ represents a chromatogram of $\mathrm{NaCl}$ solution treated under the same conditions as in Fig. 2a. The HA standard used in our study was a sodium salt. Since CAD is a quasi-universal detector, it is likely that sodium and chloride ion peaks would show up in the chromatograms. The respective sodium and chloride ion peaks are visible in Fig. 2b. Figure 2c shows the chromatogram of an acid hydrolyzed HA standard. The chromatogram indicates the presence of GlcN, GlcA, sodium, chloride and minor amounts of GlcNAc. The unknown peak is likely to be a disaccharide unit of GlcNAc and GlcA. Figure $2 d$ shows the chromatogram of an acid hydrolyzed HA serum sample. The contents of the sample in Fig. $2 d$ are identical to the HA standard indicating its authenticity. This HILIC-CAD method is easily adaptable to other similar carbohydrate-based therapeutics. The method is easy to execute and saves time compared to other laborious techniques involving derivatization. The entire monosaccharide composition analysis (hydrolysis of samples and HPLC analysis) using our method can be completed within $3 \mathrm{~h}$. This is a significant improvement compared to other traditional methods involving derivatization which can take $>24 \mathrm{~h}$.
The homogeneity of HA samples were also analyzed by HPSEC-CAD. Separation of HA standards of varying molecular weights is shown in Fig. 3a. Figure 3b, c are representative HPSEC chromatograms of HA supplements. The sample in Fig. 3b is a polymer predominantly composed of a single peak. The shape of the peak in HPSEC is often indicative of the homogeneity of the sample. The non-symmetrical peak shape in Fig. $3 \mathrm{~b}$ indicates the non-homogeneous nature of the sample. Figure $3 c$ contains two non-symmetrical peaks indicating a heterogeneous sample. The difference in molecular size and purity can have significant impact in terms of biological efficacy. HPSEC-CAD offers an easy way to check for homogeneity and size of polysaccharides.

\section{Conclusion}

This study demonstrated the utility of HPLC-CAD as a multipurpose analytical tool for $\mathrm{QC}$ analysis of carbohydrates. A HILIC-CAD method was developed for separation and detection of common uronic acids, amino sugars and select neutral sugars without derivatization. This method is an extension of other HILIC-CAD methods [9] for neutral sugar analysis. The HPLC-CAD platform can serve as a cheaper and simpler alternative to existing methods for composition analysis. Furthermore, the HPLC-CAD can be used to perform HPSEC experiments to analyze homogeneity and molecular weight of carbohydrate polymers.

\section{Limitations}

The platform can only be used as a QC tool for known carbohydrate samples. Analysis of unknown samples can be difficult. Since CAD is a semi-universal detector, unwanted impurities owing to hydrolysis

\footnotetext{
(See figure on next page.)

Fig. 2 Monosaccharide composition of HA samples by HILIC-CAD. Authentic standards and commercially purchased HA samples were hydrolyzed by 2 M TFA for $2 \mathrm{~h}$ before composition analysis by the HILIC-CAD gradient method. a Represents a chromatogram of GICNAc and GICA standards. The degradation of GlcNAc into GlcN is evident from the chromatogram. $\mathbf{b}$ Is a representative chromatogram of NaCl solution. The sodium and chloride ion peaks are visible in the chromatogram. c Shows a chromatogram of a hydrolyzed HA standard. The Cl- $\mathrm{GlcNAc}^{-} \mathrm{GlCN}_{\mathrm{C}} \mathrm{Na}^{+}$and GlcA peaks can be seen along with an unknown peak $\left.{ }^{* *}\right)$. The unknown peak is speculated to be a disaccharide repeating unit of $\mathrm{HA}$. $\mathbf{d}$ Is a representative chromatogram of a hydrolyzed HA serum sample. The composition of $\mathbf{d}$ is identical to $\mathbf{c}$ indicating that it is an authentic product
} 
Gosh and Kline BMC Res Notes

(2019) 12:268

Page 5 of 7
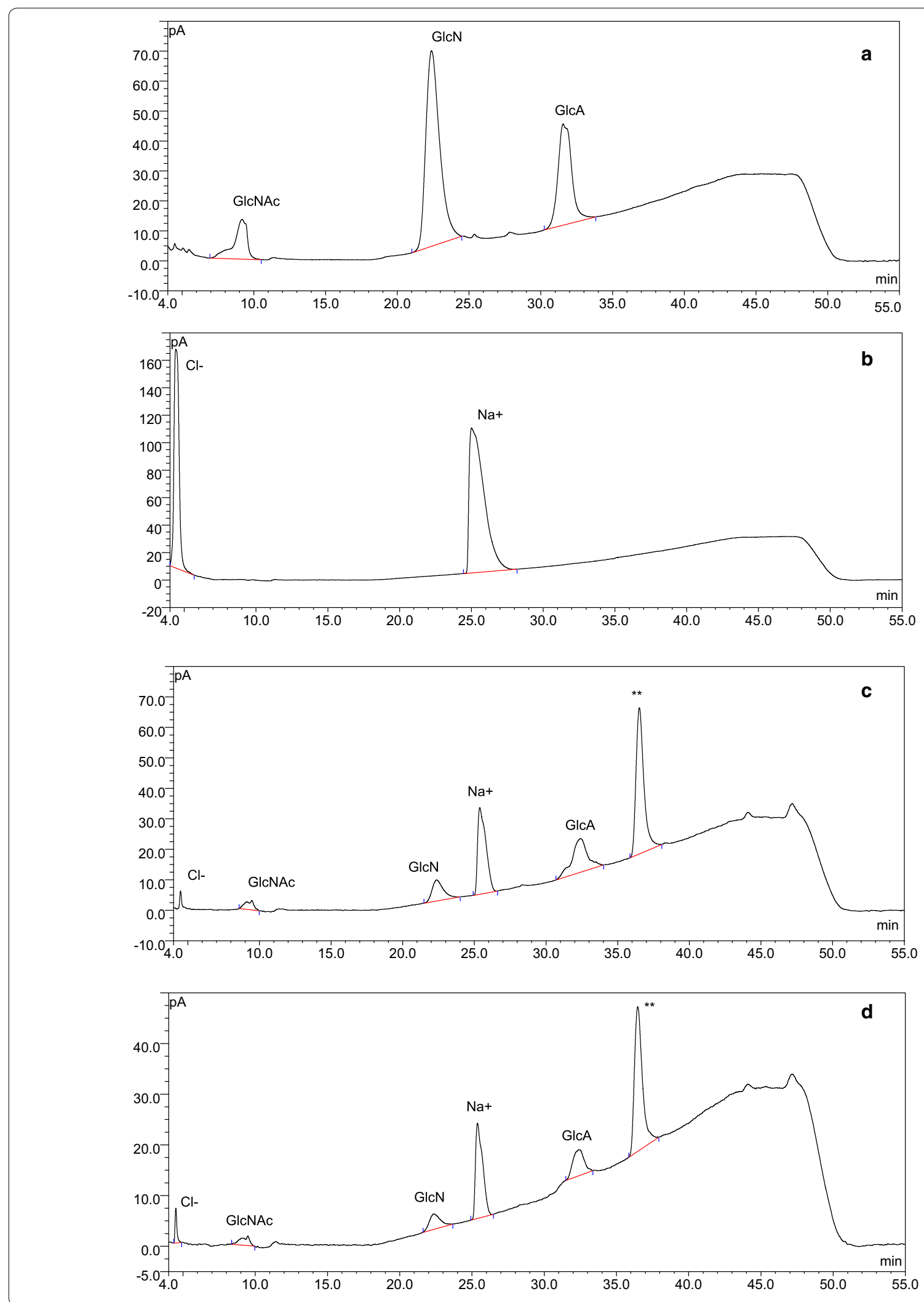

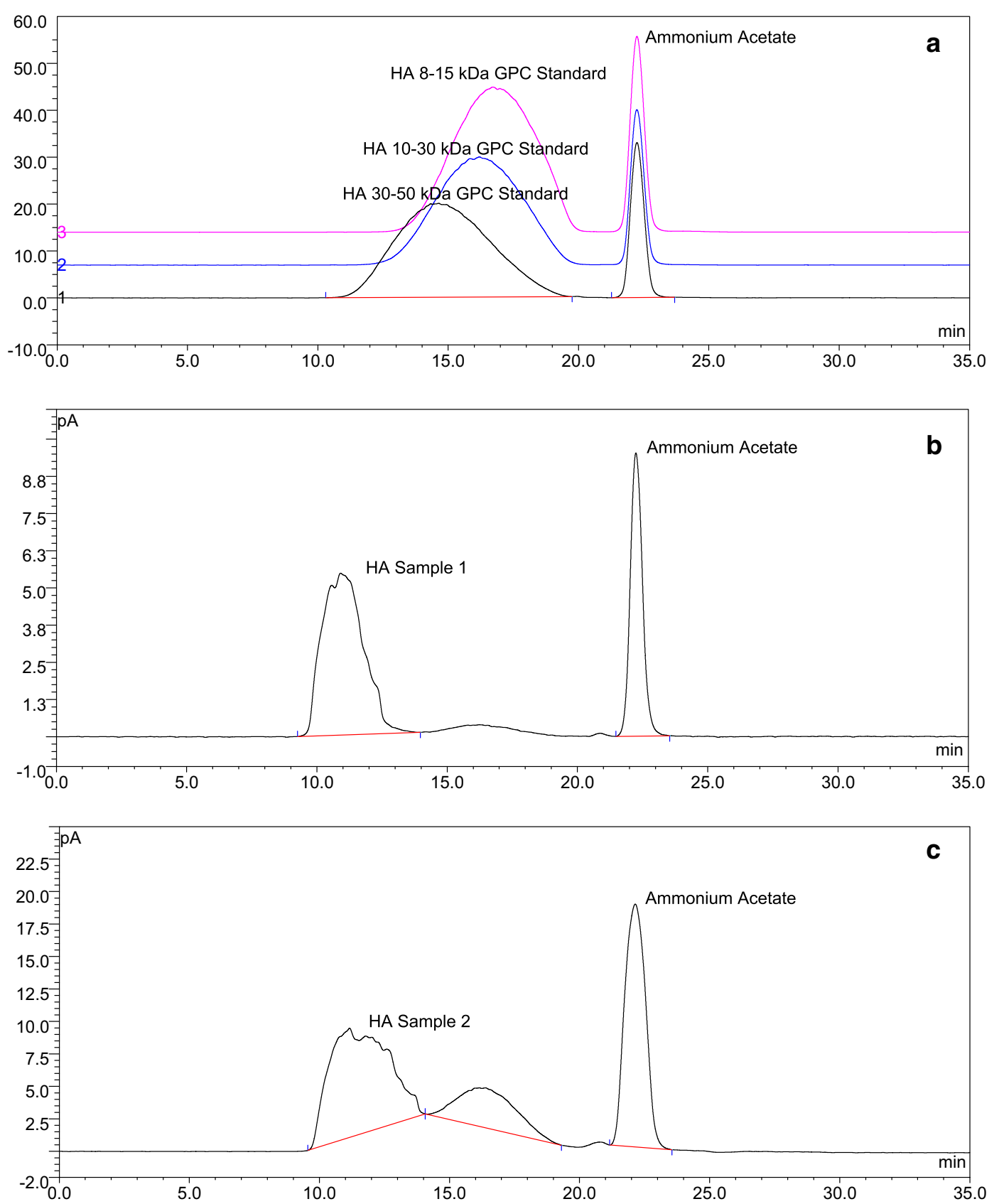

Fig. 3 Purity and homogeneity analysis of HA samples by HPSEC-CAD. a Shows the overlay of HPSEC chromatograms of HA GPC standards of varying molecular weights (pink: 8-15 kDa; blue: 10-30 kDa; black: 30-50 kDa). b, c Represent HPSEC chromatograms of commercially available HA products. Although $\mathbf{b}$ is predominantly composed of a single peak, the shape of the peak indicates that it might not be a homogeneous fraction. $\mathbf{c}$ Contains two non-symmetrical peaks indicating that it is a heterogeneous sample

conditions might interfere with composition analysis of unknown samples. Also, the HILIC-CAD method proposed in this study cannot separate all neutral sugar residues such as glucose and galactose. Our method, which separates most common acidic and amino sugars, must be used in complementation with other reported neutral sugar separation methods for complete composition analysis. 


\section{Additional file}

Additional file 1: Table S1. Linearity, LOD, LOQ and precision of the proposed HILIC-CAD method. Figure S1. Effects of column temperature on chromatographic separation. Figure S2. Effects of tertiary amine TEA on chromatographic separation. Figure $\mathbf{S 3}$. Effects of ammonium acetate on chromatographic separation.

\section{Abbreviations}

QC: quality control; GC-MS: gas chromatography-mass spectrometry; HPLC: high performance liquid chromatography; HPAEC-PAD: high performance anion exchange chromatography-pulsed amphoteric detection; HILIC: hydrophilic interaction liquid chromatography; ELSD: evaporative light scattering detector; MS: mass spectrometer; RID: refractive index detector; CAD: charged aerosol detector; SEC: size exclusion chromatography; GlCN: D-glucosamine; GalN: D-galactosamine; GlcNAc: N-acetyl-D-glucosamine; GlCA: D-glucuronic acid; GalA: D-galacturonic acid; Neu5Ac: N-acetyl-neuraminic acid; LIdoA: L-iduronic acid; LFuc: L-fucose; Gal: D-galactose; Man: D-mannose; LRha: L-rhamnose; HA: hyaluronic acid; TFA: trifluoroacetic acid; TEA: triethylamine; LOD: limit of detection; LOQ: limit of quantification; RSD: relative standard deviation; HPSEC: high performance size exclusion chromatography; GPC: gel permeation chromatography.

\section{Acknowledgements}

We would like to thank the Dr. Elliot Altman (Director, TCBMR) for supporting this research.

\section{Authors' contributions}

RG: conceptualization and study design, data collection, data analysis, manuscript preparation. PK: conceptualization and study design, supervision of project, manuscript preparation. Both authors read and approved the manuscript.

\section{Funding}

RG was funded by the Molecular Biosciences PhD program at Middle Tennessee State University and the Tennessee Center for Botanical Medicine Research (TCBMR). The funders had no role in study design, data collection, data analysis and preparation of manuscript.

\section{Availability of data and materials}

Data will be available upon request.

\section{Ethics approval and consent to participate}

Not applicable.

\section{Consent to publication}

Not applicable.

\section{Competing interests}

The authors declare that they have no competing interests.

Received: 21 March 2019 Accepted: 3 May 2019

Published online: 14 May 2019

\section{References}

1. Li S, Wu D, Lv G, Zhao J. Carbohydrate analysis in herbal glycomics. Trends Anal Chem. 2013;52:155-69.

2. Ikegami T, Horie K, Saad N, Hosoya K, Fiehn O, Tanaka N. Highly efficient analysis of underivatized carbohydrates using monolithic-silica-based capillary hydrophilic interaction (HILIC) HPLC. Anal Bioanal Chem. 2008;391:2533-42.

3. Ruiz-Matute Al, Hernández-Hernández O, Rodríguez-Sánchez S, Sanz ML, Martínez-Castro I. Derivatization of carbohydrates for GC and GC-MS analyses. J Chromatogr B Analyt Technol Biomed Life Sci. 2011;879:1226-40.

4. Yang X, Zhao Y, Wang Q, Wang H, Mei Q. Analysis of the monosaccharide components in Angelica polysaccharides by high performance liquid chromatography. Anal Sci. 2005;21:1177-80.
5. Zhang Z, Khan NM, Nunez KM, Chess EK, Szabo CM. Complete monosaccharide analysis by high-performance anion-exchange chromatography with pulsed amperometric detection. Anal Chem. 2012;84:4104-10.

6. Mariño K, Bones J, Kattla JJ, Rudd PM. A systematic approach to protein glycosylation analysis: a path through the maze. Nat Chem Biol. 2010;6:713-23

7. Karlsson G, Winge S, Sandberg H. Separation of monosaccharides by hydrophilic interaction chromatography with evaporative light scattering detection. J Chromatogr A. 2005:1092:246-9.

8. Lowenthal MS, Kilpatrick EL, Phinney KW. Separation of monosaccharides hydrolyzed from glycoproteins without the need for derivatization. Anal Bioanal Chem. 2015:407:5453-62.

9. Yan J, Shi S, Wang H, Liu R, Li N, Chen Y, Wang S. Neutral monosaccharide composition analysis of plant-derived oligo- and polysaccharides by high performance liquid chromatography. Carbohydr Polym. 2016:136:1273-80

10. Godin B, Agneessens R, Gerin PA, Delcarte J. Composition of structural carbohydrates in biomass: precision of a liquid chromatography method using a neutral detergent extraction and a charged aerosol detector. Talanta. 2011:85:2014-26

11. Dixon RW, Peterson DS. Development and testing of a detection method for liquid chromatography based on aerosol charging. Anal Chem. 2002;74:2930-7.

12. Hu DJ, Cheong KL, Zhao J, Li SP. Chromatography in characterization of polysaccharides from medicinal plants and fungi. J Sep Sci. 2013;36:1-19.

13. Ligor M, Studzinska S, Horna A, Buszewski B. Corona-charged aerosol detection: an analytical approach. Crit Rev Anal Chem. 2013:43:64-78.

14. Kou D, Manius G, Zhan S, Chokshi HP. Size exclusion chromatography with Corona charged aerosol detector for the analysis of polyethylene glycol polymer. J Chromatogr A. 2009;1216:5424-8.

15. Chen QL, Chen YJ, Zhou SS, Yip KM, Xu J, Chen HB, Zhao ZZ. Laser microdissection hyphenated with high performance gel permeation chromatography-charged aerosol detector and ultra performance liquid chromatography-triple quadrupole mass spectrometry for histochemical analysis of polysaccharides in herbal medicine: Ginseng, a case study. Int J Biol Macromol. 2018;107:332-42.

16. Li J, Hu D, Zong W, Lv G, Zhao J, Li S. Determination of inulin-type fructooligosaccharides in edible plants by high-performance liquid chromatography with charged aerosol detector. J Agric Food Chem. 2014:62:7707-13.

17. Khoomrung S, Chumnanpuen P, Jansa-Ard S, Ståhlman M, Nookaew I, Borén J, Nielsen J. Rapid quantification of yeast lipid using microwaveassisted total lipid extraction and HPLC-CAD. Anal Chem. 2013;85:4912-9.

18. Tanaka H, Zhou $X$, Masayoshi O. Characterization of a novel diol column for high-performance liquid chromatography. J Chromatogr A. 2003:987:119-25.

19. Necas J, Bartosikova L, Brauner P, Kolar J. Hyaluronic acid (hyaluronan): a review. Vet Med. 2008;53:397-411

\section{Publisher's Note}

Springer Nature remains neutral with regard to jurisdictional claims in published maps and institutional affiliations.

Ready to submit your research? Choose BMC and benefit from:

- fast, convenient online submission

- thorough peer review by experienced researchers in your field

- rapid publication on acceptance

- support for research data, including large and complex data types

- gold Open Access which fosters wider collaboration and increased citations

- maximum visibility for your research: over $100 \mathrm{M}$ website views per year

At $\mathrm{BMC}$, research is always in progress.

Learn more biomedcentral.com/submissions 